\title{
Perfil laboral de migrantes mexicanos deportados e inserción laboral en México
}

\author{
JESÚS JAVIER PEÑA MUÑOZ*
}

RESUMEN: Presentamos el perfil laboral de los mexicanos deportados de Estados Unidos y analizamos las implicaciones para su inserción laboral en México. Basamos nuestro reporte en información de la Encuesta sobre Migración en la Frontera Norte de México (Emif), correspondiente a 2014. Los datos dibujan un perfil laboral de hombres jóvenes con algún nivel de secundaria cursado, sin conocimientos del idioma inglés, con experiencia laboral en el sector agrícola, en la construcción y en establecimientos de preparación y venta de alimentos. Reconocer la diversidad interna de este flujo, en especial en relación con el tiempo de residencia en Estados Unidos, es importante para diseñar políticas dedicadas a estos migrantes.

PALABRAS CLAVE: repatriados, inserción laboral, perfil laboral, México, Estados Unidos.

* Investigador de El Colegio de la Frontera Norte, sede Ciudad Juárez, México. 
ABSTRACT: Using data from the Emif for 2014, we profile men and women of Mexican nationality who were deported to Mexico by U.S. authorities to analyze their labor qualifications and economic insertion in Mexico. The data presents a labor force composed of young males, with twelve years of education, who do not speak English, and with work experience in agriculture, construction and the food and beverage industry. Recognizing the internal diversity of these migrants, especially in regards to time of residence in the United States, is crucial to design public policy aimed at their economic integration.

KEY WORDS: deportees, labor qualifications, economic insertion, Mexico, United States. 


\section{INTRODUCCIÓN}

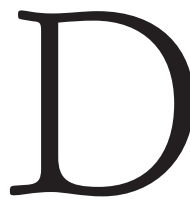

urante 2014, un promedio de 23 mil mexicanos al mes fueron deportados tanto del interior como de la franja fronteriza sur del territorio estadounidense. Entre enero y agosto de ese año, 142,482 de estos repatriados aceptaron apoyos de programas federales (UPM, 2014). Los apoyos incluyen ayuda inmediata, como atención médica, alimentación, llamadas telefónicas y ayuda para transportarse a sus lugares de origen, así como asistencia para reinsertarse en México al sector educativo o al mercado laboral. Entre estos apoyos, el empleo, siendo fuente importante de cohesión social y bienestar individual, debe ser uno de los pilares de la política pública y de los programas de apoyo orientados a combinar seguridad humana, desarrollo integral e inclusión social; también debe ser la base de la reinserción socioeconómica de migrantes deportados. El desarrollo de mecanismos institucionales eficientes de inserción laboral comienza por conocer el perfil laboral de dichos migrantes.

El presente trabajo tiene como objetivo principal presentar el perfil laboral de hombres y mujeres de nacionalidad mexicana que fueron deportados de Estados Unidos y analizar las implicaciones para su inserción laboral en México. Por perfil laboral nos referimos a educación, entrenamiento, conocimientos, experiencia de trabajo en México y en Estados Unidos y las características demográficas mencionadas relevantes en vacantes de empleo. Este reporte se basa en información de la Encuesta sobre Migración en la Frontera Norte de México (Emif) correspondiente a 2014. Durante más de 20 años la Emif Norte ha cubierto este flujo migratorio en diversos puntos de la frontera México-Estados Unidos, así como en otros puntos de llegada a México, lo que la convierte en una de las fuentes principales de información para el análisis. La información y las conclusiones de este reporte son especialmente importantes para los programas y las políticas dedicadas atender este flujo. Tal es el caso del programa Repatriados Trabajando, cuyo objetivo general es apoyar económicamente a connacionales repatriados que buscan empleo. Se aprovecha el perfil laboral para cubrir las necesidades de 
mano de obra en los lugares de origen o residencia en México y ofrecer alternativas a la migración a Estados Unidos.

La primera sección de este trabajo está dedicada a las características demográficas relevantes para el empleo, como género, edad, lugar de origen y escolaridad. A continuación, el lector encontrará una sección sobre el nivel de conocimiento del idioma inglés, seguida por una tercera sección dedicada a la experiencia laboral en México y en Estados Unidos. Finalizamos con una sección sobre entrenamiento y capacitan laboral y un apartado de conclusiones.

\section{Características demográficas}

Hasta finales de octubre de 2014, la Emif Norte captó 5,228 migrantes mexicanos deportados que arribaron a México por vía terrestre o aérea. Para adentrarnos en el análisis de este grupo, debemos recordar que, como otros tipos de migrantes, este flujo presenta variaciones internas. Primero, tenemos que atender a las posibles diferencias entre los migrantes deportados después de residir en Estados Unidos por varios años y los que fueron aprehendidos en el momento de cruzar la frontera o al poco tiempo de residir en Estados Unidos. ${ }^{1}$ Asimismo, es necesario tener en cuenta la circularidad de la migración mexicana hacia Estados Unidos. Como nos ha mostrado la investigación en el tema, estamos ante migrantes que pueden permanecer y trabajar en dicho país por largos periodos, pero que conceptualmente su lugar de residencia es México. Lo anterior influye en los perfiles y metas laborales, ya que muchos deportados entienden la condición laboral en relación con esta dinámica circular. Igual de importante es distinguir entre el flujo y la migración de retorno voluntario. Estos flujos no sólo muestran diferencias en los motivos para retornar a México, sino también en las cuestiones que influyen

${ }^{1}$ Durante el segundo trimestre de 2014, entre los encuestados que declaran tener su lugar de residencia en México, predominan quienes estuvieron de un día a menos de un mes en el país del norte. Representan 60.2 por ciento de este flujo. Entre los que definen su lugar de residencia en Estados Unidos, 88.1 por ciento contaba con tres o más años de residencia en esa nación.

$170 \frac{\text { PRIMER SEMESTRE } 2015}{\text { MIGRACIÓN Y DESARROLLO NÚM. } 24}$ 
en el perfil laboral, como tiempo de estancia en Estados Unidos, experiencia laboral y patrimonio en el momento de regresar.

Respecto al diseño de programas y políticas públicas de inserción, el retorno voluntario ofrece mayores alternativas de planeación que el retorno por deportación. Debemos tomar en cuenta estas diferencias al diseñar programas de inserción laboral para estos flujos.

Sexo

Con base en lo registrado por la Emif Norte en años anteriores, sabemos que el flujo de deportados se caracteriza por una selectividad de acuerdo con el sexo. Del total de casos de repatriación entre mayores de 18 años registrados por las autoridades migratorias mexicanas, 156,893 fueron hombres y 16,869 mujeres. En cambio, entre menores de 18 años, fueron 8,576 hombres y 1,599 mujeres. $^{2}$ Los datos de la Emif 2014 reafirman esta selectividad con 87.7 por ciento de hombres. ${ }^{3}$ Lo anterior representa un aumento de casi 4 por ciento en relación con lo registrado en 2014. Las vacantes publicadas en los periódicos de ofertas de empleo del Servicio Nacional de Empleo ligadas al programa Repatriados Trabajando no hacen distinción por el sexo. Por ello, habríamos de suponer que el sexo del candidato no es un factor decisivo en su posibilidad de obtener empleo. ${ }^{4}$ Sin embargo, es importante asentar

${ }^{2}$ Las diferentes fuentes de información estadística sobre migración mexicana nos muestran un aumento en el número de mujeres. No obstante, la llamada «feminización» de la migración mexicana se presenta con mayor claridad en otro tipo de migrantes y en otros destinos; por ejemplo, la migración calificada a Canadá y a Alemania.

${ }^{3}$ Como punto de comparación podemos remontarnos a los datos de la Emif de 2004, que muestran una participación promedio de 87.7 de hombres y de 15.3 de mujeres, según cifras de la Emif.

${ }^{4}$ No obstante, en las vacantes publicadas en listados colocados en la osne en Ciudad Juárez, Chihuahua, las vacantes de empleo especifican el sexo del candidato. Otros requisitos que aparecen en algunas vacantes y que no se mencionan en los periódicos se refieren a la apariencia física del candidato. Por lo general, se pide no tener tatuajes visibles o una buena presentación. 
que estamos ante una potencial fuerza laboral compuesta casi por completo por hombres.

$$
\text { GRÁFICA } 1
$$

Composición del flujo de deportados por sexo

$$
\text { Hombre }
$$

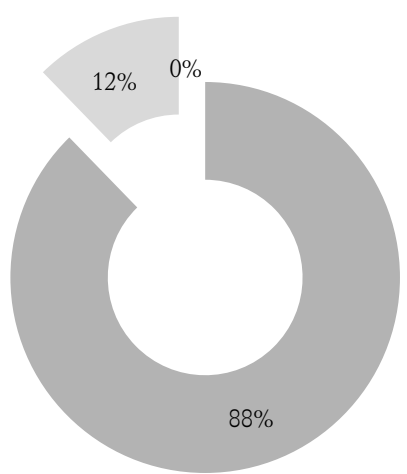

Fuente: Elaboración propia con datos de la Emif Norte (2014).

\section{Edad}

Respecto de la edad, los datos de la Emif Norte arrojan una moda de 20 años y una media de 29.7 años. El 87 por ciento tiene entre 15 y 40 años. Este flujo migratorio continúa dominado por jóvenes como lo ha sido desde hace décadas. Se trata de individuos cuyas experiencias migratorias comienzan a temprana edad. Emigran jóvenes a Estados Unidos y regresan jóvenes a México. Por delante poseen una considerable etapa productiva laboral. Por eso el empleo debe ser una de sus prioridades (y necesidades). 
TABLA 1

Estructura del flujo de deportados por edad, 2014

\begin{tabular}{lccc}
\hline GRUPO DE EDAD & ABSOLUTOS & PORCENTAJES & PORCENTAJE ACUMULADO \\
\hline 15 a 20 años & 10,819 & 15.2 & 16.8 \\
21 a 25 años & 14,575 & 22.7 & 39.4 \\
26 a 30 años & 12,316 & 19.1 & 58.5 \\
31 a 35 años & 10,851 & 16.9 & 75.3 \\
36 a 40 años & 7,559 & 11.8 & 87.0 \\
41 a 45 años & 5,218 & 8.1 & 95.1 \\
46 a 50 años & 1,929 & 3.1 & 98.1 \\
51 a 55 años & 898 & 1.3 & 99.5 \\
56 o más & 330 & 0.5 & 100.0 \\
Total & 5,228 & 100.0 & \\
\hline
\end{tabular}

Fuente: Elaboración con datos de la Emif Norte (2014).

Lugar de origen o residencia

Los datos de la Emif muestran que 76.5 por ciento de los deportados reside en sus lugares de nacimiento. Durante 2014, los seis principales lugares de nacimiento de los deportados fueron Oaxaca (11.6 por ciento), Michoacán (10.1 por ciento), Guerrero (7.8 por ciento), Puebla (7.6 por ciento) y Guanajuato (6.0 por ciento). De tal modo, un primer paso es analizar el perfil laboral de dicho flujo en relación con la oferta laboral de sus lugares de origen, dado que éstos serían los destinos más probables donde buscarían trabajo. No obstante, el regreso al lugar de origen puede no ser la mejor opción para algunos deportados. La frontera norte, los grandes centros urbanos, así como el trabajo temporal en los puntos de deportación en la espera de oportunidades para regresar a Estados Unidos son preferidos al lugar de origen, cuyos mercados laborales sufren las limitaciones surgidas en gran parte de la dinámica misma de la migración circular. 


\section{Escolaridad}

El 43.2 por ciento de los deportados captados por la Emif Norte en 2014 tiene una escolaridad a nivel secundaria, seguida por la primaria con 28.6 por ciento. Junto con la preparatoria, estos niveles abarcan 92 por ciento de los devueltos. El fenómeno anterior sugiere que la mayoría cuenta con habilidades básicas de escritura y lectura en español, así como de cálculo y matemáticas.

TABLA 2

Último año de escuela aprobada (2014)

\begin{tabular}{lrrr}
\hline \multicolumn{1}{c}{ NIVEL } & ABSOLUTOS & PORCENTAJE & PORCENTAJE ACUMULADO \\
\hline Ninguno & 1,099 & 1.7 & 1.7 \\
Primaria & 18,456 & 28.6 & 30.3 \\
Secundaria & 30,711 & 47.6 & 77.9 \\
Preparatoria & 9,526 & 14.8 & 92.7 \\
Escuela técnica con secundaria & 214 & .3 & 93.0 \\
Normal & 32 & .0 & 93.1 \\
Profesional & 765 & 1.2 & 94.3 \\
Elementary & 81 & .1 & 94.4 \\
High School & 3,201 & 5.0 & 99.4 \\
College & 344 & .5 & 99.9 \\
Maestría/ Masters & 12 & .0 & 99.9 \\
No sabe & 39 & .1 & 100.0 \\
No especificado & 14 & .0 & 100.0 \\
Total & 6,4493 & 100.0 & \\
\hline
\end{tabular}

Fuente: Elaboración con datos de la Emif Norte (2013).

Otro dato importante sobre la escolaridad de los deportados es si cursaron algún nivel de escolaridad en Estados Unidos. Como podemos verificar en la gráfica 2, solamente 5.6 por ciento cursó el último año de escuela en el mencionado país. ${ }^{5}$

5 Esto representa un aumento en relación con 4 por ciento reportado por la Emif Norte en 2013. Dicho aumento se explica en parte por el incremento de deportaciones de personas que han estado viviendo en Estados Unidos por periodos prolongados.

$174 \frac{\text { PRIMER SEMESTRE } 2015}{\text { MIGRACIÓN Y DESARROLLO NÚM. } 24}$ 


\section{GRÁFICA 2}

País donde se aprobó el último año de escolaridad (2014)

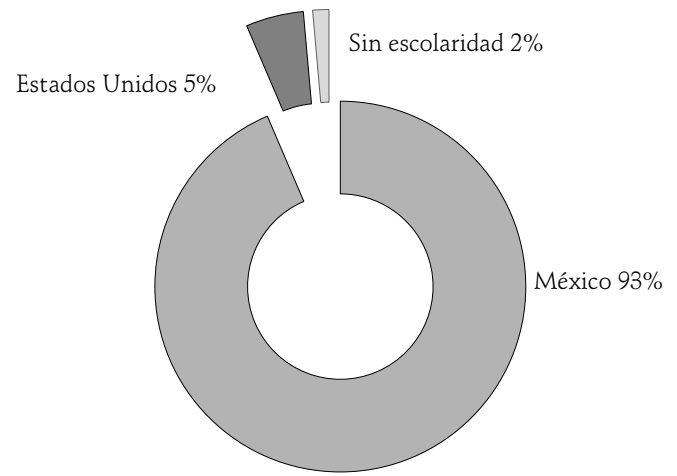

Fuente: Elaboración propia con datos de la Emif Norte (2014).

\section{GRÁFICA 3}

Conocimiento del idioma inglés

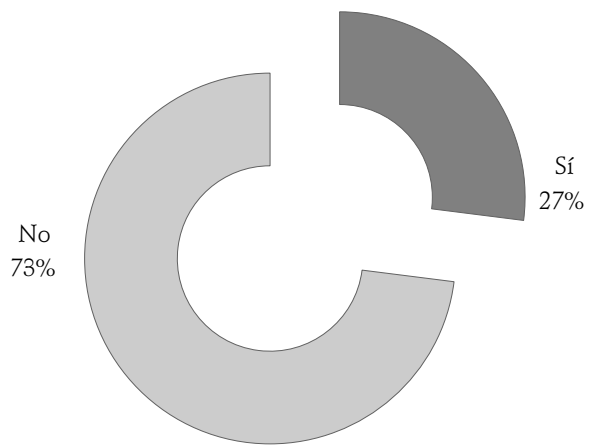

Fuente: Elaboración propia con datos de la Emif Norte (2014). 


\section{GRÁFICA 4}

Nivel en el que hablan inglés (2014)

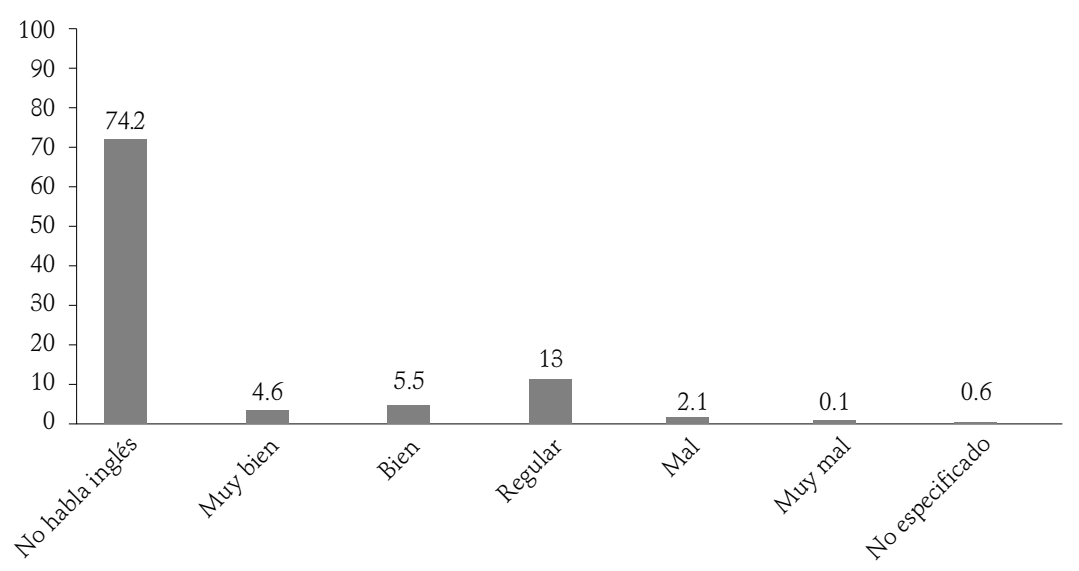

Fuente: Elaboración propia con base en datos de la Emif Norte (2014).

TABLA 3

Conocimiento para leer y escribir en inglés y español

¿SABE ESCRIBIR EN ESPAÑOL, INGLÉS O EN AMBOS IDIOMAS?

\begin{tabular}{|c|c|c|c|c|c|}
\hline & & FRECUENCIA & PORCENTAJE & $\begin{array}{l}\text { PORCENTAIE } \\
\text { VÁLIDO }\end{array}$ & $\begin{array}{l}\text { PORCENTAJE } \\
\text { ACUMULADO }\end{array}$ \\
\hline \multirow[t]{6}{*}{ Válidos } & $\begin{array}{l}\text { No sabe leer y } \\
\text { escribir }\end{array}$ & 1,182 & 1.8 & 1.8 & 1.8 \\
\hline & Español & 55,409 & 85.9 & 85.9 & 87.7 \\
\hline & Inglés & 389 & .6 & .6 & 88.4 \\
\hline & Ambos idiomas & 7,482 & 11.6 & 11.6 & 100.0 \\
\hline & No sabe & 30 & .0 & .0 & 100.0 \\
\hline & Total & 64,493 & 100.0 & 100.0 & \\
\hline
\end{tabular}

Fuente: Emif Norte, octubre de 2014.

$176 \frac{\text { PRIMER SEMESTRE } 2015}{\text { MIGRACIÓN Y DESARROLLO NÚM. } 24}$ 


\section{IDIOMA INGLÉS}

Relacionado con su educación y tiempo de residencia en Estados Unidos, el conocimiento del idioma inglés luce útil en el momento de buscar empleo en ciertos sectores. No obstante, sólo 25.8 por ciento de los encuestados declaró hablar inglés.

Entre quienes reportaron hablar inglés, 10 por ciento describió su manejo del idioma como muy bien o bien. La mayoría considera que lo habla de manera regular a muy mal.

En cuanto a los conocimientos de escritura y lectura, 85.9 por ciento sabe leer y escribir en español. En la tabla 3 se aprecia que son pocos los encuestados que saben leer y escribir en inglés y español, pero con 11.6 por ciento es mayor al porcentaje que no sabe leer ni escribir en ningún idioma.

\section{EXPERIENCIA LABORAL}

Las tablas 4 y 5 muestran la experiencia laboral en los últimos 30 días a la deportación. Cuando analizamos la experiencia laboral de estos migrantes, debemos prestar atención al tiempo de residencia en Estados Unidos. En un extremo, tenemos a los migrantes detenidos por las autoridades migratorias estadounidenses en el intento de cruzar la frontera. En el otro extremo, se hallan los migrantes deportados después de haber vivido en Estados Unidos por años. Otro grupo intermedio está formado por migrantes cuya estancia en Estados Unidos ha sido de manera intermitente e incluso han sido deportados en más de una ocasión. Respecto al primer grupo cuya experiencia laboral ha sido solamente en México, encontramos que menos de 40 por ciento ha trabajado en los últimos 30 días en su lugar de residencia. Estos bajos porcentajes se deben a la falta de empleo en los lugares de residencia (uno de los motivos principales de la migración) y al hecho de haber pasado parte de ese tiempo en el intento por llegar a Estados Unidos.

Entre quienes lograron cruzar a Estados Unidos y permanecer en ese país por lo menos un año, 46.3 por ciento trabajó allí en los 30 días previos a la 
TABLA 4

Experiencia laboral en los últimos 30 días en el lugar de residencia DURANTE LOS ÚLTIMOS 30 DíAS OUE ESTUVO, i̇TRABAJÓ USTED EN ESE LUGAR O EN ALGÚN LUGAR CERCANO

\begin{tabular}{|c|c|c|c|c|c|}
\hline & & FRECUENCIA & PORCENTAJE & $\begin{array}{l}\text { PORCENTAJE } \\
\text { VÁLIDO }\end{array}$ & $\begin{array}{l}\text { PORCENTAJE } \\
\text { ACUMULADO }\end{array}$ \\
\hline \multirow[t]{6}{*}{ Válidos } & $\begin{array}{l}\text { Naicó en otro } \\
\text { país }\end{array}$ & 36 & .1 & .1 & .1 \\
\hline & $\begin{array}{l}\text { Vive en EUA o } \\
\text { vive en México } \\
\text { y permaneció } \\
\text { un año o más } \\
\text { en EUA }\end{array}$ & 17,591 & 27.3 & 27.3 & 27.3 \\
\hline & $\begin{array}{l}\text { No ha trabaja- } \\
\text { do donde vive o } \\
\text { lugar cercano }\end{array}$ & 9,243 & 14.3 & 14.3 & 41.7 \\
\hline & Sí & 22,597 & 35.0 & 35.0 & 76.7 \\
\hline & No & 15,025 & 23.3 & 23.3 & 100.0 \\
\hline & Total & 64,493 & 100.0 & 100.0 & \\
\hline
\end{tabular}

Fuente: Emif Norte (2014).

TABLA 5

Experiencia laboral en los últimos 30 días en Estados Unidos

DURANTE LOS ÚLTIMOS 30 DÍAS QUE ESTUVO EN ESTADOS UNIDOS, i̇TRABAJÓ USTED?

\begin{tabular}{|c|c|c|c|c|c|}
\hline & & FRECUENCIA & PORCENTAJE & $\begin{array}{l}\text { PORCENTAJE } \\
\text { VÁLIDO }\end{array}$ & $\begin{array}{l}\text { PORCENTAJE } \\
\text { ACUMULADO }\end{array}$ \\
\hline \multirow{6}{*}{ Válidos } & Nació en otro país & 36 & .1 & .1 & .1 \\
\hline & $\begin{array}{l}\text { Vive en México y permane- } \\
\text { ció menos de un año en EUA }\end{array}$ & 46,865 & 72.7 & 72.7 & 72.7 \\
\hline & No ha trabajado en EUA & 1,732 & 2.7 & 2.7 & 75.4 \\
\hline & Sí & 8,148 & 12.6 & 12.6 & 88.0 \\
\hline & No & 7,711 & 12.0 & 12.0 & 100.0 \\
\hline & Total & 64,493 & 100.0 & 100.0 & \\
\hline
\end{tabular}

Fuente: Emif Norte (2014).

$178 \frac{\text { PRIMER SEMESTRE } 2015}{\text { MIGRACIÓN Y DESARROLLO NÚM. } 24}$ 
TABLA 6

Experiencia laboral en el lugar de origen en los últimos 30 días antes de la deportación

\begin{tabular}{|c|c|c|}
\hline OFICIO O PROFESIÓN & ABSOLUTOS & $\begin{array}{l}\text { PORCENTAIE DEL TOTAL QUE SÍ TRABAJÓ } \\
\text { EN LOS ÚLTIMOS } 30 \text { MESES }\end{array}$ \\
\hline Actividades agrícolas & 8,304 & 36.7 \\
\hline $\begin{array}{l}\text { Albañilería y otros trabajos en la edificación de cons- } \\
\text { trucciones }\end{array}$ & 2,554 & 11.30 \\
\hline Trabajadores de apoyo en la construcción y plomería & 1,886 & 8.3 \\
\hline Conductores de transporte terrestre con motor & 1,882 & 8.3 \\
\hline Trabajador de apoyo en la industria & 1,153 & 5.1 \\
\hline $\begin{array}{l}\text { Preparación y servicio de alimentos y bebidas en esta- } \\
\text { blecimientos }\end{array}$ & 924 & 4.1 \\
\hline Comerciantes en establecimientos & 660 & 2.9 \\
\hline $\begin{array}{l}\text { Herreros, cerrajeros, joyeros y artesanos en la elabo- } \\
\text { ración de productos de metal }\end{array}$ & 636 & 2.8 \\
\hline Empleados de ventas y vendedores por teléfono & 607 & 2.6 \\
\hline $\begin{array}{l}\text { Trabajadores de limpieza, recamaristas, mozos de } \\
\text { limpieza y limpiadores de calzado }\end{array}$ & 459 & 2.0 \\
\hline $\begin{array}{l}\text { Yeseros, instaladores de pisos, impermeabilizante, } \\
\text { vidrio, plomeros y pintores }\end{array}$ & 293 & 1.3 \\
\hline
\end{tabular}

Fuente: Elaboración propia con datos de la Emif Norte (2014).

TABLA 7

Giro productivo del lugar de trabajo

\begin{tabular}{lcc}
\hline \multicolumn{1}{c}{ SECTOR } & ABSOLUTOS & PORCENTAJE DEL TOTAL ENCUESTADO \\
\hline Agricultura & 7,965 & 12.4 \\
Edificación & 4,410 & 6.8 \\
Servicios de reparación y mantenimiento & 1,152 & 1.8 \\
Transporte terrestre de pasajeros excepto & 1,195 & 1.9 \\
ferrocarril & & 1.7 \\
Comercio al por menor de alimentos, be- & 1,126 & 1.3 \\
bidas y tabaco & 848 & \\
Industria alimentaria & & \\
\hline
\end{tabular}

Fuente: Elaboración propia con datos de la Emif Norte (2014). 
deportación. Es una cifra baja para un flujo considerado primordialmente laboral. Lo anterior nos sugiere que una parte de esta movilidad está motivada por objetivos no laborales y por una falta de puestos de trabajo en los sectores de colocación de migración indocumentada. Esta última resulta una tendencia que, de 2007 a la fecha, se muestra como uno de los principales factores en los flujos de retorno voluntario.

En cuanto a los oficios o profesiones comunes entre quienes reportaron haber trabajado en los últimos 30 días en sus lugares de residencia en México, en los resultados hasta octubre de 2014, la encuesta de la Emif Norte registra gran diversidad, con más de 90 oficios o profesiones diferentes. Sin embargo, los trabajos relacionados con la agricultura y la construcción logran concentrar casi la mitad de los casos. La tabla 6 muestra las 11 profesiones u oficios que registraron el mayor número de menciones.

Si distinguimos la experiencia laboral por sector o industria, encontramos que el sector agrícola y el de construcción continúan siendo uno de los nichos laborales principales entre los que tuvieron en México su último empleo previo a la deportación. A pesar de que el perfil de la migración indocumentada se ha ido diversificando en favor de una migración urbana, una parte considerable de los migrantes que componen este flujo continúa realizando actividades laborales en el sector agrícola.

Ahora veamos qué oficios o profesiones son las más comunes entre quienes reportaron haber trabajado en los últimos 30 días en Estados Unidos. Hallamos también gran variedad de oficios y profesiones. No obstante, es posible observar una concentración en profesiones relacionadas con la construcción y con establecimientos de venta de alimentos. El sector de la construcción es uno de los principales empleadores de estos migrantes en ambos lados de la frontera. Por el contrario, en Estados Unidos el sector agrícola pierde relevancia ante trabajos en establecimientos de venta de comida, como restaurantes. La tabla 8 muestra los 11 sectores laborales con mayor número de menciones por parte de los encuestados.

$\mathbf{1 8 0} \frac{\text { PRIMER SEMESTRE } 2015}{\text { MIGRACIÓN Y DESARROLLO NÚM. } 24}$ 
TABLA 8

Experiencia laboral en Estados Unidos en los últimos 30 días

\begin{tabular}{|c|c|c|}
\hline OFICIO O PROFESIÓN & ABSOLUTOS & $\begin{array}{l}\text { PORCENTAJE DEL TOTAL } \\
\text { QUE SÍ TRABAJÓ EN LOS ÚLTIMOS } \\
30 \text { DÍAS EN ESTADOS UNIDOS }\end{array}$ \\
\hline Albañilería y otros trabajos en la edificación de construcciones & 1,900 & 23.3 \\
\hline $\begin{array}{l}\text { Preparación y servicio de alimentos y bebidas en estableci- } \\
\text { mientos }\end{array}$ & 1,110 & 13.6 \\
\hline $\begin{array}{l}\text { Yeseros, instaladores de pisos, climas, impermeabilizante, vi- } \\
\text { drio, plomeros y pintores }\end{array}$ & 874 & 10.7 \\
\hline Jardineros & 803 & 9.8 \\
\hline Actividades agrícolas & 785 & 9.6 \\
\hline $\begin{array}{l}\text { Mecánicos y técnicos en mantenimiento y reparación de equi- } \\
\text { po mecánico, vehículos de motor, instrumentos industriales }\end{array}$ & 462 & 5.6 \\
\hline $\begin{array}{l}\text { Artesanos y trabajadores en la elaboración de productos de } \\
\text { madera }\end{array}$ & 185 & 2.3 \\
\hline $\begin{array}{l}\text { Técnicos en electrónica y de equipo de telecomunicaciones y } \\
\text { electromecánicos }\end{array}$ & 150 & 1.8 \\
\hline Moldeadores, soldadores, hojalateros y pintores de metales & 136 & 1.6 \\
\hline Cajeros, cobradores y pagadores & 128 & 1.5 \\
\hline $\begin{array}{l}\text { Supervisores de trabajadores en la extracción, albañiles y en } \\
\text { acabados de construcción }\end{array}$ & 122 & 1.4 \\
\hline
\end{tabular}

Fuente: Elaboración propia con datos de la Emif Norte (2014).

\section{Capacitación laboral}

Entre quienes trabajaron en los últimos 30 días en sus lugares de origen, es poco común que hayan recibido algún tipo de capacitación laboral. Como muestra la tabla 9, solamente 5.2 por ciento del total captado por la Emif Norte hasta octubre de 2014 reportó haber recibido algún curso de capacitación por parte de la empresa donde trabajaba antes de emigrar.

Algo similar observamos en relación con las prestaciones laborales y los beneficios. 5.4 por ciento reportó haber tenido alguna prestación en el puesto laboral anterior a la migración. En conjunto, estas cifras presentan la imagen de un mercado laboral de origen precario, con pocas posibilidades de desarrollo y de estabilidad económica, condiciones que hacen de la migración internacional indocumentada una opción forzada para muchos. 
TABLA 9

\section{Capacitación en trabajo anterior}

¿RECiBIÓ ALGÚN CURSO DE CAPACITACIÓN EN LA EMPRESA QUE TRABAJÓ?

\begin{tabular}{|c|c|c|c|c|c|}
\hline & & FRECUENCIA & PORCENTAJE & $\begin{array}{l}\text { PORCENTAJE } \\
\text { VÁLIDO }\end{array}$ & $\begin{array}{l}\text { PORCENTAJE } \\
\text { ACUMULADO }\end{array}$ \\
\hline \multirow[t]{8}{*}{ Válidos } & Naicó en otro país & 36 & .1 & .1 & .1 \\
\hline & $\begin{array}{l}\text { Vive en EUA o vive en } \\
\text { México y permaneció un } \\
\text { año o más en EUA }\end{array}$ & 17,591 & 27.3 & 27.3 & 27.3 \\
\hline & $\begin{array}{l}\text { No trabajó en los últimos } \\
30 \text { días donde vive por } \\
\text { diferentes razones }\end{array}$ & 3,421 & 5.3 & 5.3 & 32.6 \\
\hline & $\begin{array}{l}\text { No trabajó, pero buscó } \\
\text { trabajo }\end{array}$ & 10,345 & 16.0 & 16.0 & 48.7 \\
\hline & $\begin{array}{l}\text { No ha trabajado donde } \\
\text { vive o lugar cercano }\end{array}$ & 9,243 & 14.3 & 14.3 & 63.0 \\
\hline & Sí & & 5.2 & 5.2 & 68.2 \\
\hline & No & 3,343 & 31.8 & 31.8 & 100.0 \\
\hline & Total & 64,493 & 100.0 & 100.0 & \\
\hline
\end{tabular}

Fuente: Emif Norte (2014).

TABLA 10

Prestaciones en trabajo anterior

EN EL TRABAJO QUE DESEMEPNó, iTENÍA ALGUNA PRESTACIÓN O BENEFICIO?

\begin{tabular}{|c|c|c|c|c|c|}
\hline & & FRECUENCIA & PORCENTAJE & $\begin{array}{l}\text { PORCENTAJE } \\
\text { VÁLIDO }\end{array}$ & $\begin{array}{l}\text { PORCENTAJE } \\
\text { ACUMULADO }\end{array}$ \\
\hline \multirow[t]{8}{*}{ Válidos } & Nació en otro país & 36 & .1 & .1 & .1 \\
\hline & $\begin{array}{l}\text { Vive en EUA o vive en } \\
\text { México y permaneció un } \\
\text { año o más en EUA }\end{array}$ & 17,591 & 27.3 & 27.3 & 27.3 \\
\hline & $\begin{array}{l}\text { No trabajó en los últimos } \\
30 \text { días donde vive por } \\
\text { diferentes razones }\end{array}$ & 3,421 & 5.3 & 5.3 & 32.6 \\
\hline & $\begin{array}{l}\text { No trabajó, pero buscó } \\
\text { trabajo }\end{array}$ & 10,345 & 16.0 & 16.0 & 48.7 \\
\hline & $\begin{array}{l}\text { No ha trabajado donde } \\
\text { vive o lugar cercano }\end{array}$ & 9,243 & 14.3 & 14.3 & 63.0 \\
\hline & Sí & & 5.4 & 5.4 & 68.2 \\
\hline & No & 3,460 & 31.6 & 31.6 & 100.0 \\
\hline & Total & 64,493 & 100.0 & 100.0 & \\
\hline
\end{tabular}

Fuente: Emif Norte (2014). 


\section{Conclusiones}

Los flujos de deportados representan una fuerza de trabajo compuesta de hombres jóvenes que han cursado algún nivel de secundaria o preparatoria en México, por lo que saben leer y escribir en español. Hablan poco inglés y no lo leen o escriben. Han formado sus conocimientos, habilidades y experiencia laboral en el campo y en la construcción, sin opciones de capacitación ni prestaciones o beneficios. El tiempo de estancia en Estados Unidos inyecta cierta variación en el perfil laboral. Sin embargo, esta variación se basa más en el tipo de sector laboral que en habilidades adquiridas, como el conocimiento del idioma inglés. El sector agrícola, importante empleador en algunos lugares de origen, parece haber alcanzado un límite en su capacidad de absorber migración laboral indocumentada. En su lugar, otros sectores, como la venta de alimentos y profesiones relacionadas con la construcción, emplean un alto porcentaje de estos migrantes. Este último es el sector laboral que podríamos considerar como un nicho para este tipo de migración. Por ello, este sector, a través del sector público y privado, luce como vía importante para la integración laboral de los migrantes. Por medio de programas públicos, se podría lograr la inserción laboral de los repatriados, quienes serían ocupados en actividades de construcción y mantenimiento de infraestructura pública en sus lugares de origen.

El desarrollo de mecanismos institucionales eficientes de inserción laboral comienza por tener claro que los deportados conforman un tipo particular de migración de retorno que debe ser atendida de manera particular mediante programas especializados, como el programa social Repatriados Trabajando, un programa de la Secretaría del Trabajo y Prestación Social (STPS) que ofrece apoyos económicos y de canalización a migrantes deportados para que encuentren empleo en sus lugares de residencia en México. El vínculo interinstitucional de este programa con otros y con instituciones públicas, así como con el sector privado, es clave para aumentar las opciones de inserción laboral ofrecidas por dicho programa. El aprovechamiento de nuevas habilidades y de experiencia laboral adquiridos por los migrantes implica dotar a los programas de inserción laboral, de herramientas de orientación, 
apoyo económico, estudio de mercado, educación y capacitación que agreguen el autoempleo a las alternativas de inserción laboral.

Un reto importante para el diseño institucional de mecanismos de inserción laboral de la migración de retorno consiste en los cambios demográficos que se proyectan para estos flujos. Si bien por varias décadas estos flujos han estado compuestos principalmente de migración joven en edad laboral, dentro de 20 años podremos estar ante el retorno, ya sea por deportación o voluntario, de una migración envejecida que busca en México alternativas de retiro. Aun cuando para el retorno por deportación este cambio luce más tardado y en menor proporción, debemos comenzar a tomar en cuenta lo anterior en las políticas de inserción laboral de estos migrantes.

\section{Bibliografía}

El Colegio de la Frontera Norte (2014), «Encuesta sobre Migración en la Frontera Norte de México», en www.colef.mx/emif (consultado el 10 de noviembre de 2014)

Secretaría de Gobernación, Unidad de Política Migratoria (2014) <<FALTA TíTULO DEL DOCUMENTO CONSULTADO>>, http://www.politicamigratoria. gob.mx/es_mx/SEGOB/Estadística (consultado el 21 de noviembre de 2014). 